\title{
Shaping White Light Through Electroluminescent Fully Organic Coupled Microcavities
}

\author{
By Marco Mazzeo,* Fabio della Sala, Fabrizio Mariano, Giovanna Melcarne, \\ Stefania D'Agostino, Yu Duan, Roberto Cingolani, and Giuseppe Gigli
}

White organic light emitting diodes (white-OLEDs) have shown the potential to replace commercial sources for lighting applications because of their high efficiency and superior color quality. The most conventional architectures used to maximize the performances of such devices consist in the employment of three kinds of emitting phosphorescent or fluorescent molecules and modified glass substrates for the optical management of light outcoupling. ${ }^{[1-5]}$ Maximum power efficiencies larger than $70 \mathrm{~lm} \mathrm{~W} \mathrm{~W}^{-1}$ and color rendering index (CRI) values higher than 80 have been recently achieved by means of phosphorescent materials, ${ }^{[1,2]}$ while power efficiencies larger than $20 \mathrm{~lm} \mathrm{~W} \mathrm{~W}^{-1}$ have been obtained with more stable fluorescent compounds. ${ }^{[3]}$

In both cases external outcoupling systems have been used. Although very promising, these approaches are still based on the use of expensive ${ }^{[6]}$ and relatively low conductive indium tin oxide (ITO) substrates, ${ }^{[7]}$ thus leading to high fabrication costs and poor color emission homogeneity on large area devices. ${ }^{[8]}$

More importantly, since ITO is in short supply, ${ }^{[6]}$ a massproduction of white-OLEDs for a global conversion of current lighting systems is not realistic keeping the conventional architectures. In addition, in these current layouts the optimization of white-OLED performances is bound to a proper selection of active molecules without any possibilities to control their intrinsic emission properties. For all these reasons, the development of an ITO-free low-cost technology, which is also able to manage the optical properties of the emitters, could create new opportunities for lighting applications. Although many techniques have been developed to manage the light emission patterns of active molecules, through the control of photonic mode density (PMD) distribution, they resulted in limited power efficiency enhancement, poor control of emission

[*] Dr. M. Mazzeo, Dr. F. Mariano, Dr. G. Melcarne University of Salento

Scuola Superiore ISUFI, 73100, Lecce (Italy)

E-mail:marco.mazzeo@unile.it

Dr. F. della Sala, Dr. S. D’Agostino, Dr. Y. Duan

National Nanotechnology Laboratory

CNR-ISTITUTO NANOSCIENZE U.O.S. LECCE

Via Arnesano, 16, 73100, Lecce (Italy)

Prof. R. Cingolani,

Italian Institute of Technology (IIT)

Via Morego, 30, 16163, Genova (Italy)

Prof. G. Gigli

Department of Innovation Engineering

University of Salento

Campus Universitario, 73100, Lecce (Italy)

DOI: 10.1002/adma.201001631 shape, and/or unacceptably high fabrication cost. ${ }^{\left[{ }^{-12]}\right.}$ Here we report an innovative high performance ITO-free white-OLED architecture based on the coupling of two organic microcavities constituted by only thermally evaporated metallic and organic layers. In the proposed structure, named a coupled-microcavity white device (CM), two cavities are coupled through a thin highreflective metal layer, to result in the generation of two electromagnetic modes sustained by the whole cavity. If white light emitting molecules are inserted properly into the structure as active layer, and managing the PMD around the organic emitters, all the device characteristics can be improved with respect to ITO-based devices. With our approach, the color emission, the outcoupling efficiency, and the CRI can be simultaneously optimized in the same cavity structure. This opens new pathways to fabricate a novel class of very cheap, high color quality, and ITO-free devices for the next generation of "plastic-light" sources.

The CM-OLEDs are constituted of two cavities (C1 and C2) each made by two metallic mirrors (silver or aluminum) separated by sub-micrometric organic stacks. The common metallic layer works as the coupler of the two cavities and the degree of coupling is determined by its thickness. The structure is realized by depositing on a transparent substrate (glass in this work but plastic foils can be used) the following layer sequence: glass/ $\mathrm{AgB} / \mathrm{OS} 1 / \mathrm{AgM} / \mathrm{OS} 2 / \mathrm{AgT}$, where $\mathrm{AgB}$ is a semitransparent silver bottom layer, OS1 the first active organic stack where the emitters are placed, AgM the middle coupler silver layer, OS2 a second passive organic stack, and AgT a thick reflective silver layer (see inset of Figure 1a). The anode and the cathode are $\mathrm{AgB}$ and AgM respectively. White light is emitted through the semitransparent bottom electrode. OS2 is filled with a standard organic material which should have a high energy gap to avoid the absorption of light. Compared to tandem technology, ${ }^{[13]}$ in which both the stacked cells are active, the presented structure is based on a single active cell (OS1) and a passive one (OS2). Because of this intrinsic configuration the applied voltage is not increased as in the tandem structures and, therefore, an improvement of current efficiency will result in an improvement of power efficiency as well.

The operation principle of the coupled-cavities ${ }^{[14]}$ is analogous to that of two mechanical harmonic oscillators coupled by a spring, with OS1 and OS2 acting as oscillators and the common AgM metallic layer as the coupler (spring). The degree of coupling (elastic constant of the spring) is determined by the AgM thickness/transmittance. The use of a thin AgM layer with high transmittance results in two strongly coupled cavities generating in-phase and out-of-phase electromagnetic modes, whose wavelengths are dependent on the AgM thickness itself. 

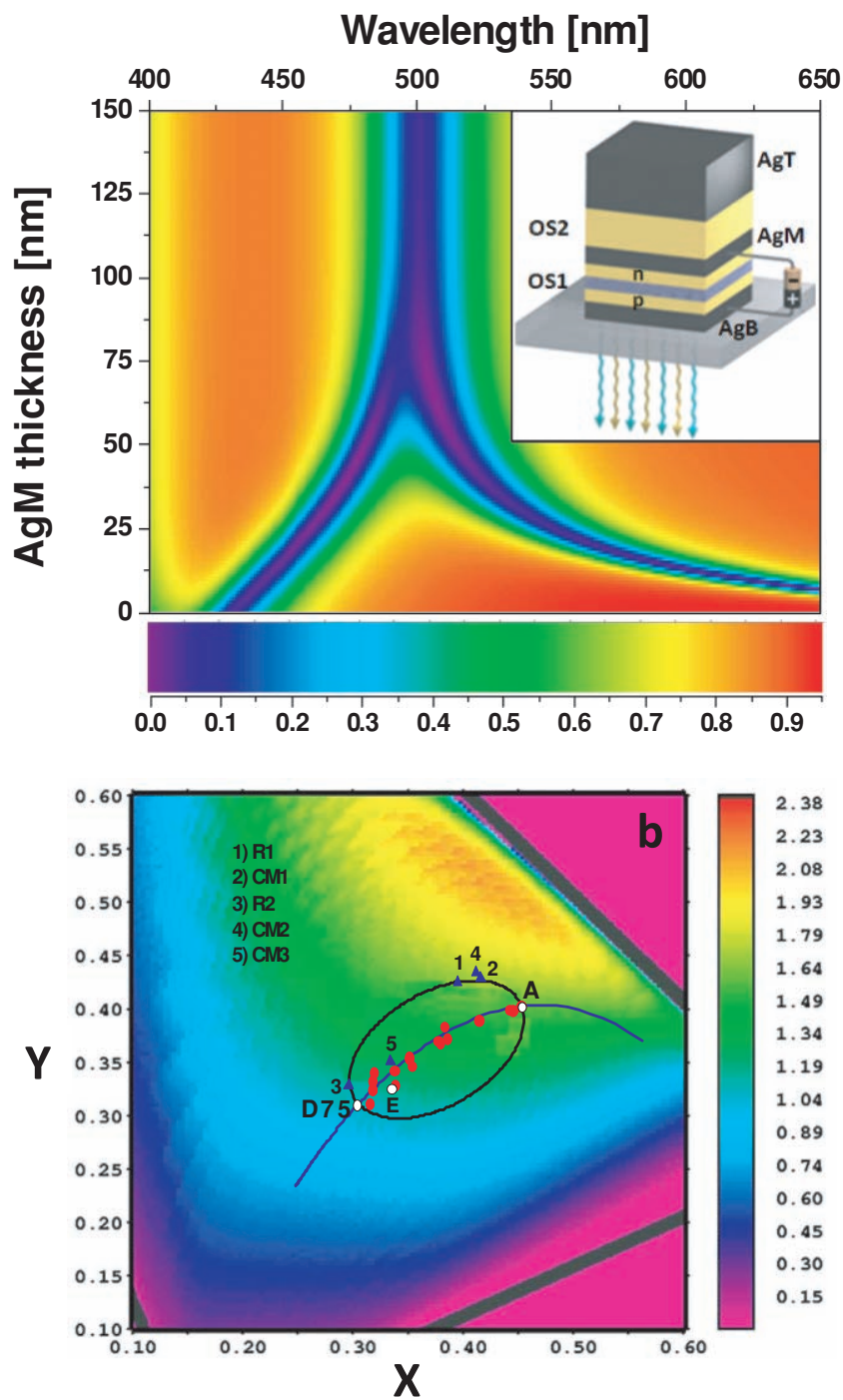

Figure 1. a) Computed reflectivity spectrum as a function of $\mathrm{AgM}$ thickness for a $\mathrm{CM}$ white device $(\mathrm{OS} 1=\mathrm{OS} 2=85 \mathrm{~nm}, \mathrm{AgB}=20 \mathrm{~nm})$. The inset is a schematic view of the $\mathrm{CM}$ configuration used in this work, exclusively made of metallic and organic layers. The blue layer indicates the emitting layer. $\mathrm{p}$ and $\mathrm{n}$ represent the hole- and the electron-conductive sides of the OLED. b) Maximum forward luminance (color scale) obtained with the ideal coupled cavity (ICM) device for each point of the CIE coordinates, normalized to that of the best ideal reference structure (IR1). The white ellipse in the CIE diagram is defined in the text. Red dots represent all the main commercial F1-12 lamps used currently in the lighting market, while the blue curve represents the Planckian (blackbody) locus. The reported color coordinates (blue triangles) are those of the real devices fabricated in this work using fluorescent compounds, as reported in the experimental session of the main text.

The basic equations of a general inorganic coupled cavity are reported by Stanley et al. ${ }^{[14]}$ and can be adapted to our layers based only on metals and organic compounds.

This behavior can be observed in the reflectivity map reported in Figure 1a and has been obtained for a symmetric cavity (equal OS1 and OS2 thickness) by means of transmission-matrix electromagnetic calculations. Here the AgM thickness varies in the range $0-150 \mathrm{~nm}$. As expected, as it is reduced below the cutoff value (around $60 \mathrm{~nm}$ ), the electromagnetic fields overlap each other and generate two resonant wavelengths longer and shorter, respectively, than the resonant wavelength of the uncoupled-cavity (high AgM thickness). More importantly, by varying the AgM thickness and the relative lengths of the two cavities, the position of the two wavelengths can be tuned over all the visible range. Therefore, once the materials to employ as white emitters are established, the cavity can be designed in order to amplify just the modes that correspond to the peakwavelengths of the white emission spectrum and/or to change completely the spectral shape. This is mainly because of the concentration of PMD around the selected wavelengths.

In order to investigate the potential of CMs in white-OLED technology, we have computed, by a numerical electromagnetic approach (see Experimental Section), the relative forward luminance and the CIE color coordinates of a reference device (glass $/ \mathrm{ITO} / \mathrm{p} / \mathrm{n} / \mathrm{AgT}$ ) with a standard ITO electrode and of a $\mathrm{CM}$ structure (glass/AgB/p/n/AgM/OS2/AgT). In both structures we assumed the emitting layer to be constituted by a dimensionless layer of isotropically distributed emitting dipoles located at the interface between the two organic layers, named $\mathrm{p}$ and $\mathrm{n}$ in the inset of Figure 1a, and with a white emission spectrum constant over all the wavelengths. For all the organic layers ( $\mathrm{p}, \mathrm{n}, \mathrm{OS} 2$ ) we assumed a constant and non-dispersive refractive index ${ }^{[15]} n=1.77$. These ideal configurations allow the effect of the cavity structure to be decoupled from the peculiar characteristics of the materials used. The computed color coordinates and luminance can be simultaneously optimized just by changing the geometrical parameters of the cavities.

In Figure $1 b$, for each point of the CIE diagram, we reported (color scale) the forward luminance intensity of the best ideal coupled-microcavity (ICM) structure normalized to the maximum forward luminance achievable by the ideal cavity-less reference configuration (IR1). Provided that all dipoles have a broad emission spectrum, from the reported map it follows that almost all the points of the CIE diagram can be reached, including all the white colors around/on the Planckian curve. This opens the possibility to fabricate for the first time a full panchromatic OLED. Moreover, because of the concentration of PMD in the microcavity resonance modes, the spontaneous emission of the emitters can be enhanced ${ }^{[16,17]}$ to result in an improvement of the relative luminance up to $108 \%$ in the yellowish-white color region $(0.40<X<0.45 ; 0.40<Y<0.45)$. In the white-region ellipse (Figure 1c), which includes all the white color coordinates reasonably near the Planckian-curve and those of the most common commercial white light sources (red points), the CM structures still provide a remarkable lightemission increase up to $70 \%$.

The ideal case has been compared with real prototypes of white-OLEDs based on two complementary color fluorescent materials, ${ }^{[18]}$ namely the blue (B) light-emitting 4,4-bis2,2-diphenylvinyl-1,1-spirobiphenyl (spiro-DPVBi) and the yellow (Y) light-emitting 5,6,11,12-tetraphenylnaphthacene (rubrene) compounds, (see Figure 2a for chemical structures). In particular the emitting layer consists of $15 \mathrm{~nm}$ of 4,4,4-tris- $N$-2-naphthyl- $N$-phenylamino triphenylamine (NPB) doped with $0.9 \%$ of rubrene covered by $5 \mathrm{~nm}$ of spiro-DPVBi. Other systems based on three primary color phosphorescent, 


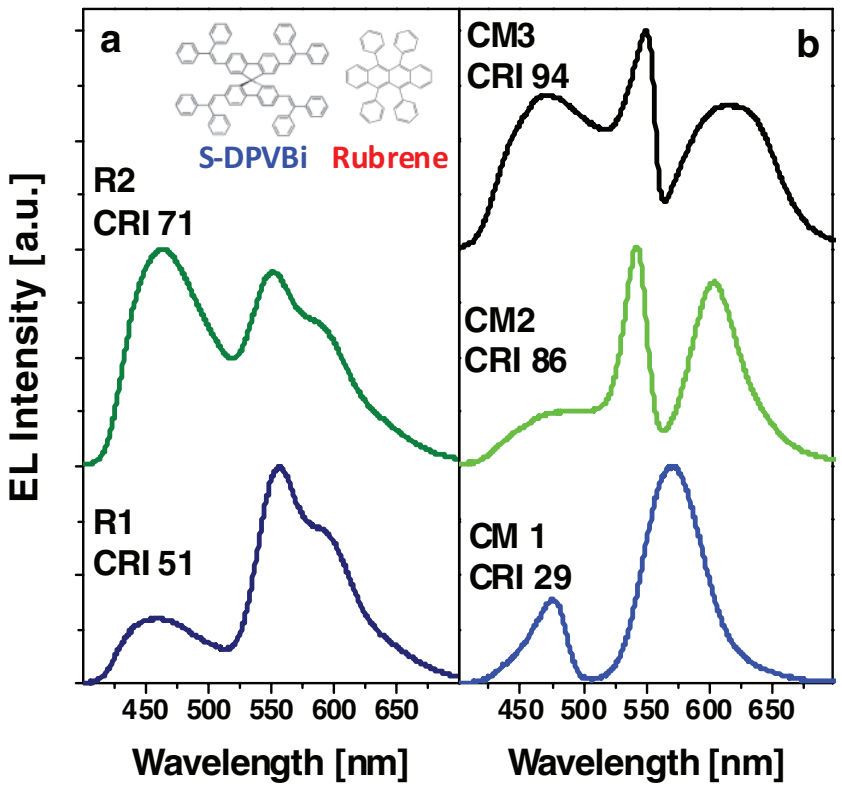

Figure 2. a) Reference devices EL spectra. b) Cavity devices EL spectra. Blue lines correspond to the spectra shown by the series 1 . Green lines correspond to the spectra of series 2 . The black line is the high CRI device spectrum (series 3 ). The chemical structures of the emitters employed (spiro-DPVBi and rubrene) are also shown.

fluorescent, or fluorescent/phosphorescent emitters can be selected as well, being the approach not dependent on the materials used. The structures consist of the following sequences: ITO/p/EBL/Y/B/HBL/n/AgT for the reference structures (R) and AgB/p/EBL/Y/B/HBL/n/AgM/OS2/AgT for the $C M$ devices. Here $p$ and $n$ layers are the hole and electron conductive transport layers while EBL and HBL are the electron and the hole blocking layer, respectively. The fabrication details are reported in the methods summary. We underline that silver has been used both for the cathode (AgM) and anode $(\mathrm{AgB})$, despite its poor hole injection properties. This problem has been overcome by using $p-i-n$ technology, where the $\mathrm{p}$ and $\mathrm{n}$ transport layers are heavily doped to reduce ohmic losses to a negligible level and allow for a tunnel injection of the carriers from the metal layers. ${ }^{[19]}$ An additional advantage of p-i-n structures is the decoupling of the optical properties from the electrical characteristics, thus making the device structure optimization much easier. ${ }^{[20]}$ Three series of devices have been realized with color emission inside the "white-ellipse" of Figure 1b. In the first series of devices (coupled-microcavity, CM1, and reference cavity-less, R1) the current efficiency, namely the luminance in the forward direction divided by current density, has been optimized regardless of the CRI. In the second series, both the CM and the reference structures (CM2 and R2) have been optimized fixing the angular-averaged CRI at 70, which still corresponds to an acceptable luminance. Finally, in the third series (CM3) we have maximized the CRI of the CM configuration regardless of the efficiency. For the structures and resumed performance details see Tables 1-2; for CIE color coordinates see Figure 1b (blue triangles).

In Figure 2a,b the electroluminescence (EL) spectra (blue lines) of the CM1 and R1 devices are displayed. As predicted, the two main peaks of $\mathrm{R} 1$ are selected in the CM1 structure because of management of the PMD. The CM1 architecture results in better current efficiency values than the R1 structure. An enhancement around $67 \%$, from $6.6 \mathrm{Cd} \mathrm{A}^{-1}$ in $\mathrm{R} 1$ to $11 \mathrm{Cd} \mathrm{A}^{-1}$ in $\mathrm{CM} 1$ at $1000 \mathrm{Cd} \mathrm{m}^{-2}$ (Table 2), is indeed achieved. The CM1 architecture also shows better luminance values at fixed operational voltages (Figure 3a) compared to the R1 device. For example, at $4 \mathrm{~V}$ a luminance of $9400 \mathrm{Cd}$ $\mathrm{m}^{-2}$ is achieved in the cavity-less device against $25600 \mathrm{Cd}$ $\mathrm{m}^{-2}$ obtained in the cavity structure. This strong improvement is ascribed to the cavity effect and also to the higher current density because of the presence of silver in place of ITO. Such better electro-optical performances result in higher power efficiencies (see Figure 3b), namely the total emitted light intensity, measured in lumens, normalized to the electrical power consumed to drive the device. An enhancement of around $36 \%$ at $1000 \mathrm{Cd} \mathrm{m}^{-2}$, from $11 \mathrm{~lm} \mathrm{~W}^{-1}$ (R1) to $15 \mathrm{~lm} \mathrm{~W} \mathrm{~W}^{-1}$ (CM1) is obtained. However, since we have optimized the forward luminance regardless of the CRI, this last parameter is reduced from 51 (R1) to 29 (CM1). This is caused by the lack of green light emission in the microcavity structure (Figure $2 \mathrm{~b}$ ) and a consequent poor coverage of the visible range.

This problem is overcome in the second series of devices where the microcavity structure is designed in order to provide two resonant peaks in the green and red regions, keeping also a blue-emission from spiro-DPVBi. As a consequence we obtained a spectrum showing three peaks, as in three primary color based devices, ${ }^{[1,2,21]}$ but just using two different emitters, thanks to a fine management of PMD. This results in a strong reduction of device complexity and fabrication costs and, more importantly, in the achievement of high forward CRI, as reported for CM2. Here a value as high as 86 has been obtained, similar to the best so far reported. ${ }^{[1,3,22]}$ In Figure 3c the CRI of R2 and CM2 as a function of the visual angle are reported. As expected, while a negligible angle-dependence of the CRI is found for the cavity-less structure (R2), a larger variation is

Table 1. Layer's thicknesses (in $\mathrm{nm}$ ) of the optimized structures presented in this work.

\begin{tabular}{lccccccccccc}
\hline & ITO & AgB & P & EBL & Y & B & HBL & n & AgM & OS2 & AgT \\
\hline R1-2 & 120 & 0 & 22 & 10 & 15 & 5 & 10 & 36 & 0 & 0 & 200 \\
CM1 & 0 & 18 & 22 & 10 & 15 & 5 & 10 & 36 & 28 & 84 & 200 \\
CM2 & 0 & 14 & 38 & 10 & 15 & 5 & 10 & 28 & 32 & 101 & 200 \\
CM3 & 0 & 14 & 48 & 10 & 15 & 5 & 10 & 32 & 38 & 104 & 200 \\
\hline
\end{tabular}


www.MaterialsViews.com

Table 2. Main parameters of the fabricated devices. From left to right: luminance at $4 \mathrm{~V}$; color coordinates $(X ; Y)$, voltage at $1000 \mathrm{Cd} \mathrm{m}^{-2}$, voltage at $10000 \mathrm{Cd} \mathrm{m}^{-2}$, maximum power efficiency, maximum current efficiency and forward CRI.

\begin{tabular}{|c|c|c|c|c|c|c|c|c|}
\hline & 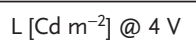 & CIE-X & CIE-Y & $V @ 1000 \mathrm{Cd} \mathrm{m}^{-2}$ & $\mathrm{~V} @ 10000 \mathrm{Cd} \mathrm{m}^{-2}$ & Pow.Eff. @ $1000 \mathrm{Cd} \mathrm{m}^{-2}$ & Curr.Eff. @ $1000 \mathrm{Cd} \mathrm{m}^{-2}$ & CRI \\
\hline $\mathrm{R} 1$ & 9400 & 0.390 & 0.430 & 2.9 & 4.1 & 11 & 6.6 & 51 \\
\hline $\mathrm{CM} 1$ & 25600 & 0.411 & 0.434 & 2.8 & 3.3 & 15 & 11 & 29 \\
\hline R2 & 7260 & 0.291 & 0.333 & 2.9 & 4.3 & 8.4 & 5.1 & 71 \\
\hline $\mathrm{CM} 2$ & 20000 & 0.407 & 0.439 & 2.9 & 3.5 & 12 & 7.4 & 86 \\
\hline $\mathrm{CM} 3$ & 4500 & 0.329 & 0.356 & 3.2 & 5.2 & 1.6 & 1.0 & 94 \\
\hline
\end{tabular}

instead observed for CM2 because of microcavity effects, the average being in any way still equal to cavity-less structure. However, the angle dependence of CRI can be strongly reduced by means of scattered structures. ${ }^{[23]}$ Unlike the typical cavity behavior, the emission intensity dependence vs the visual angle of CM2 is super-lambertian while the angular dependence of its color coordinates is kept in a range of $(0.35-0.40)$ for the $X$ coordinate and $(0.40-0.44)$ for the $Y$ coordinate. This is a similar to the $Y$ coordinate range of the reference device R2, which indicates that the color variation can be strongly minimized (see Supporting Information).

In Figure $3 \mathrm{a}, \mathrm{b}$ and in Table 2 the electro-optical performances of devices $\mathrm{R} 2$ and $\mathrm{CM} 2$ are reported. At $1000 \mathrm{Cd} \mathrm{m}^{-2}$, the microcavity device still shows a remarkable increase of current efficiency by a factor 1.45 compared to the reference cavity-less device, going from 5.1 to $7.4 \mathrm{Cd} \mathrm{A}^{-1}$. Moreover, a brightness of $10000 \mathrm{Cd} \mathrm{m}^{-2}$ is reached at $3.5 \mathrm{~V}$ in CM2, which is the lowest voltage so far reported in white-OLEDs. A maximum power efficiency value of $12.6 \mathrm{~lm} \mathrm{~W}^{-1}$ (Figure $3 \mathrm{~b}$ ) is obtained in the CM2 device, with respect to $8.4 \mathrm{~lm} \mathrm{~W}$ W $^{-1}$ shown by the reference structure, which corresponds to an enhancement of around 50\%. In addition an efficiency of $11 \mathrm{~lm} \mathrm{W^{-1 }}$ is still achieved at the remarkable luminance of $10000 \mathrm{Cd} \mathrm{m}^{-2}$, similar to the highest so far reported at this luminance using conventional ITO-based technology and without outcoupling substrates. ${ }^{[1,3]}$ In the third class of devices
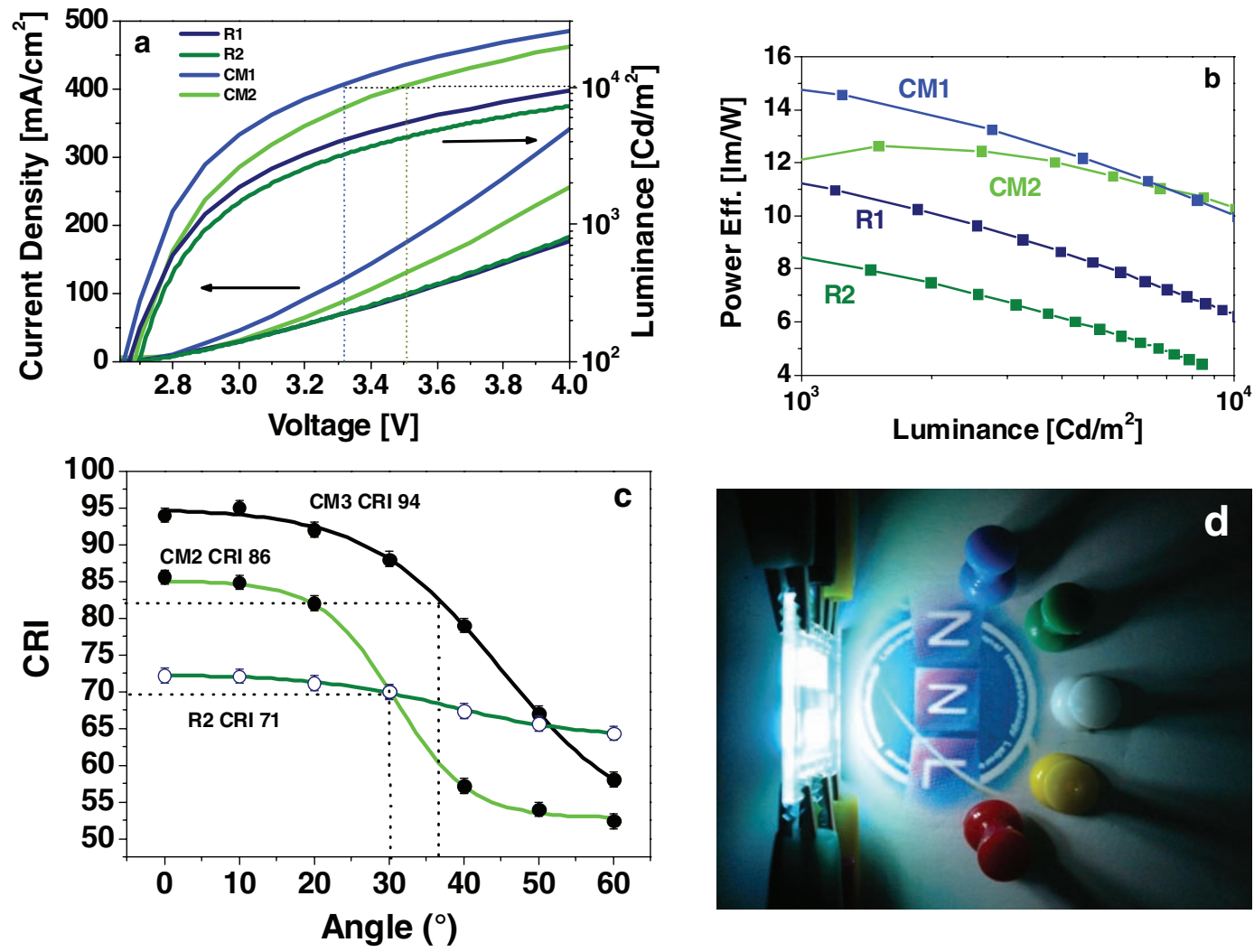

Figure 3. a) Luminance and current density curves of the device series 1 and 2. b) Power efficiency of device series 1 and 2. c) CRI data of series 2 (R2 and $\mathrm{CM} 2$ ) and of the $\mathrm{CM} 3$ device as a function of the visual angle. d) Photo of a working CM3 device at $10000 \mathrm{Cd} \mathrm{m}^{-2}$. 
(CM3) the CRI has been optimized regardless of the efficiency value. An impressive CRI of 94 in the forward direction is achieved (Figure 3c), which is similar to the highest value so far reported for conventional ITO-based white-OLEDs. ${ }^{[3]}$ The color coordinates of the CM3 structure are $X=0.329$ and $Y=0.356$, which correspond to a correlated color temperature CCT of $5650 \mathrm{~K}$, near that shown by natural day-light $(5500-6000 \mathrm{~K})$, and indicates that the white color is almost pure. The average CRI is still very high, and reaches a value of 82, even though in this case the power efficiency is strongly decreased (Table 2 and Figure 3c). Much better performances can be obtained using active materials which can approximate the ideal case discussed before. In Figure $3 \mathrm{~d}$ we report a picture of the CM3 device at $10000 \mathrm{Cd} \mathrm{m}^{-2}$. In conclusion our results show that the coupled-cavity architecture is a general approach to increase the efficiency, the luminance, the CRI, and to fully tune the emission color of any optimized OLED layer sequence, in the entire CIE white-ellipse, including the black-body curve. The approach is independent of the active materials, is compatible with other light outcoupling techniques, and it can be applied to phosphorescent emitters or hybrid fluorescent-phosphorescent compounds. The results suggest that the main problem of the end of cheap ITO can be overcome introducing this low-cost architecture based only on thermally evaporated metallic and organic layers and resulting in a novel generation of high color quality whiteOLEDs for lighting applications.

\section{Experimental Section}

Simulations: We have performed numerical electromagnetic simulations using the Emissive Thin Film Optical Simulator (ETFOS) Linux-version 1.5. ${ }^{[24]}$ For the constrained optimization of the structures we wrote a FORTRAN program coupled to the ETFOS script version.

Device and Cavity Fabrication and Characterization: The p-doped layer consists of $N, N, N^{\prime}, N^{\prime}$-tetrakis (4-methoxyphenyl)benzidine (MeO-TPD) doped with 2,3,5,6-tetrafluoro-7,7,8,8-tetracyanoquinodimethane (F4TCNQ), while the n-doped layer consists of 4,7-diphenyl-1,10phenanthroline (BPhen) doped with cesium atoms. Films of pure MeO-TPD (10 nm thick) and pure Bphen have been deposited as interlayers to avoid quenching because of the presence of dopants. In the microcavities, OS2 consists of a NPB layer. All films were deposited and prepared as reported in reference. ${ }^{[25]}$

\section{Supporting Information}

Supporting Information is available from the Wiley Online Library or from the author.

\section{Acknowledgements}

This work was partially funded by the European Research Council Starting Grant Project “DEDOM”, Grant agreement no. 207441.

Received: May 4, 2010

Published online: August 30, 2010

[1] S. Reineke, F. Lindner, G. Schwartz, N. Seidler, K. Walzer, B. Lüssem, K. Leo, Nature 2009, 459, 234.

[2] Y. Sun, S. R. Forrest, Nat. Photon. 2008, 2, 483.

[3] S. Murano, M. Burghart, J. Birnstock, P. Wellmann, M. Vehse, A. Werner, T. Canzler, T. Stübinger, G. He, M. Pfeiffer, H. Boerner, Proc. SPIE 2005, 5937, 79.

[4] T. Nakayma, K. Hiyama, K. Furukawa, H. Ohtani, Society for Information Display Digest 2007, 19, 1018.

[5] S. Moller, S. R. Forrest, J. Appl. Phys. 2002, 91, 3324.

[6] R. Baetens, B. P. Jelle, A. Gustavsen, Sol. Energy Mater. Sol. Cells, doi:10.1016/j.solmat.2009.08.021.

[7] K. Fehse, K. Walzer, K. Leo, W. Lövenich, A. Elschner, Adv. Mater. 2007, 19, 441.

[8] C. Piliego, M. Mazzeo, M. Salerno, R. Cingolani, G. Gigli, A. Moro, Appl. Phys. Lett. 2006, 89, 103514.

[9] J. Feng, T. Okamoto, Opt. Lett. 2005, 30, 2302.

[10] Y. R. Do, Y. C. Kim, Y. W. Song, Y. H. Lee, J. Appl. Phys. 2004, 96, 7629.

[11] A. Dodabalapur, L. J. Rothberg, T. M. Miller, Appl. Phys. Lett. 1994, 65, 2308.

[12] T. Shiga, H. Fujikawa, Y. Taga, J. Appl. Phys. 2003, 93, 19.

[13] T. W. Lee, T. Noh, B. K. Choi, M. S. Kim, D. W. Shin, Appl. Phys. Lett. 2008, 92, 043301.

[14] R. P. Stanley, R. Houdre, U. Oesterle, M. Ilegems, C. Weisbuch, Appl. Phys. Lett. 1994, 83, 2093.

[15] T. Y. Cho, C. L. Lin, C. C. Wu, Appl. Phys. Lett. 2006, 88, 111106.

[16] W. L. Barnes, J. Mod. Opt. 1998, 45, 661.

[17] P. T. Worthing, W. L. Barnes, J. Opt. A: Pure Appl. Opt. 1999, 1, 501.

[18] P. Van Gemmern, V. Van Elsbergen, S. P. Grabowski, H. Boerner, H.-P. Löbl, H. Becker, H. Kalisch, M. Heuken, R. H. Jansen, J. Appl. Phys. 2006, 100, 123707.

[19] K. Walzer, B. Männig, M. Pfeiffer, K. Leo, Chem. Rev. 2007, 107, 1233.

[20] R. Merheim, R. Nitche, K. Leo, Appl. Phys. Lett. 2008, 93, 043310.

[21] Z. Zhang, Q. Wang, Y. Dai, Y. Liu, L. Wang, D. MaHigh, Org. Electron. 2009, 10, 491

[22] Y. Sun, N. C. Giebink, H. Kanno, B. Ma, M. E. Thompson, S. R. Forrest, Nature 2006, 440, 908.

[23] H.-Y. Lin, J.-H. Lee, M.-K. Wei, C.-L. Dai, C.-F. Wu, Y.-H. Ho, H.-Y. Lin, T.-C. Wu, Opt. Commun. 2007, 275, 464.

[24] ETFOS version 1.5, Fluxim AG, Switzerland. www.fluxim.com

[25] Y. Duan, M. Mazzeo, V. Maiorano, F. Mariano, D. Qin, R. Cingolani, G. Gigli, Appl. Phys. Lett. 2008, 92, 113304. 